\title{
Xiaoyaosan (Tiaogan-Liqi therapy) protects peritoneal macrophages from corticosterone-induced stress by regulating the interaction between glucocorticoid receptor and ABCA1
}

\author{
Mingtai Chen ${ }^{1 \#}$, Ruolan Huang ${ }^{2 \#}$, Wenjun $\mathrm{Fu}^{3}$, Lijun Ou ${ }^{1}$, Ling Men ${ }^{4}$, Zhong Zhang ${ }^{1}$, Shudong Yang ${ }^{4}$, \\ Qiang Liu ${ }^{1}$, Jienan Luan ${ }^{1}$ \\ ${ }^{1}$ Department of Cardiovascular Disease, Shenzhen Traditional Chinese Medicine Hospital, the $4^{\text {th }}$ Clinical Medical College of Guangzhou University \\ of Chinese Medicine, Shenzhen, China; ${ }^{2}$ Department of Neurology, Shenzhen University Clinical Research Center for Neurological Diseases, \\ Shenzhen University General Hospital, Shenzhen, China; ${ }^{3}$ Centre for Integrative Medicine, School of Basic Medical Science, Guangzhou University \\ of Chinese Medicine, Guangzhou, China; ${ }^{4}$ Department of Nephrology, Shenzhen Traditional Chinese Medicine Hospital, the $4^{\text {th }}$ Clinical Medical \\ College of Guangzhou University of Chinese Medicine, Shenzhen, China \\ Contributions: (I) Conception and design: R Huang, Q Liu, Z Zhang; (II) Administrative support: S Yang; (III) Provision of study materials or patients: \\ W Fu, L Ou; (IV) Collection and assembly of data: M Chen, L Men; (V) Data analysis and interpretation: M Chen; (VI) Manuscript writing: All \\ authors; (VII) Final approval of manuscript: All authors. \\ \#These authors contributed equally to this work. \\ Correspondence to: Dr. Mingtai Chen; Prof. Qiang Liu; Prof. Jienan Luan. Department of Cardiovascular Disease, Shenzhen Traditional Chinese \\ Medicine Hospital, the $4^{\text {th }}$ Clinical Medical College of Guangzhou University of Chinese Medicine, 1 Fuhua Road, Futian District, Shenzhen, \\ China. Email: zyycardio@foxmail.com; 13802263916@139.com; luanjienan@sohu.com.
}

Background: Previous studies have reported that Xiaoyaosan (XYS), Tiaogan-Liqi therapy, has a protective function in depressive disorder, and can regulate body weight and corticosterone (CORT) level. However, little is known about the effect of XYS in treating atherosclerosis. This study aimed to explore the influence XYS on macrophage foam cell formation and related mechanism.

Methods: Rat peritoneal macrophages (PMs) were separated and stimulated with CORT and oxidized low density lipoprotein (ox-LDL). The serum was obtained from rats treated with different doses of XYS and was added into the medium for macrophages. Then, the cell activity and lipid content of PMs were measured through Cell Counting Kit-8 (CCK-8) assay and oil red staining, respectively. The expressions of glucocorticoid receptor (GR), ATP binding cassette subfamily A member 1 (ABCA1), and heat shock protein 90 (HSP90) were detected. In addition, overexpression of GR and ABCA1 was performed and the effect on XYS treatment was subsequently assessed.

Results: The CCK-8 assay showed the serum increased cell activity of CORT-induced stress PMs in a XYS dose-dependent manner. Oil red staining and enzyme-linked immunosorbent assay (ELISA) showed that the serum decreased lipids of PMs. In the XYS treated groups, HSP90 protein was decreased and protein levels of ABCA1 and GR were increased in cytoplasm, while GR protein in nucleus was decreased. Co-immunoprecipitation (Co-IP) assay indicated GR might interact with HSP90 and be involved with the function of XYS. Furthermore, overexpression of GR attenuated the protective function of XYS on CORTinduced stress in PMs, while overexpression of ABCA1 enhanced that.

Conclusions: This study denoted that XYS could protect PMs from CORT-induced stress by regulating the interaction of GR and ABCA1, which might contribute to the treatment of atherosclerosis.

Keywords: Traditional Chinese medicine; atherosclerosis; macrophages; corticosterone; Xiaoyaosan

^ ORCID: 0000-0003-4579-5559. 
Submitted Sep 10, 2020. Accepted for publication Oct 27, 2020.

doi: 10.21037/atm-20-6505

View this article at: http://dx.doi.org/10.21037/atm-20-6505

\section{Introduction}

Xiaoyaosan (XYS) is a kind of traditional Chinese medicine including Angelicae sinensis Radix [root of Angelica sinensis (Oliv) (Dang gui)], Atractylodis Macrocephalae Rhizoma [root and rhizome of Atractylodes macrocephala Koidz (Bai zhu)], Bupleuri Radix [root of Bupleurum Chinese DC (Chai bu)], Paeoniae Radix Alba [root of Paeonia lactiflora Pall (Bai shao)], Poria [fungus nucleus of Poria cocos (Schw) (Fu ling)], Menthae Haplocalycis Herba [overground parts of Mentha haplocalyx Briq (Bo he)], Zingiberis Rhizoma Recens [root and rhizome of Zingiber offcinale Rosc(Sheng jiang)] and Glycyrrbizae Radix et Rbizoma [root and rhizome of Glycyrrbiza uralensis Fish (Gan cao)] (1,2). Generally, XYS has been primarily used for the treatment of depression as monotherapy or in combination with other drugs in China $(3,4)$. Further, previous studies have shown XYS could substantially reduce the depression of gastrointestinal symptoms $(5,6)$. Xiaoyaosan has also been shown to decrease the levels of blood adrenocorticotropic hormone (ACTH) and corticosterone (CORT) (7); CORT increases serum total cholesterol (TC) and accelerates atherosclerosis (8). However, little is known about the effect of XYS on treating atherosclerosis.

Glucocorticoid (GC), a kind of endogenous steroid hormone, is a well-known regulator of the stress response and immune system (9). The function of GC is mainly mediated and limited by glucocorticoid receptors (GRs) $(10,11)$. The nuclear receptor superfamily includes GRs, which are modular proteins that normally insert in the cytoplasm and are in contact with the complex of numerous proteins such as heat shock protein 90 (HSP90) $(12,13)$. In the nucleus, GR can control the transcription of many of its target genes in multiple and highly context-specific ways. Hence, the transcriptional activity of GR is associated with stress response and metabolism regulation (14). As previous studies have indicated, GR might regulate the function of ATP-binding cassette transporter A1 (ABCA1), to control the efflux of cholesterol from macrophages $(15,16)$. There is a close correlation between ABCA1 and the level of circulating high-density lipoprotein (HDL) and accumulation of cholesteryl ester (CE) in various tissues, which are mostly distributed in macrophage-derived foam cells (17). Furthermore, it showed that ABCA1 might play a key role in the efflux of cholesterol from macrophages and peripheral cells (18). Due to the significant roles of GR and ABCA1 in macrophages, it is necessary to investigate whether XYS regulates the expression of GR and ABCA1 in macrophage foam cell formation.

In order to explore the function of XYS in macrophages, we created a CORT-induced stress model in peritoneal macrophages (PMs) and stimulated the PMs with serum after use of XYS. We found the serum could play a protective function via regulation of the expression of ABCA1 and GR. Our study might offer a new idea for the treatment of atherosclerosis. We present the following article in accordance with the ARRIVE reporting checklist (available at http://dx.doi.org/10.21037/atm-20-6505).

\section{Methods}

\section{Animals}

A total of 40 Sprague Dawley (SD) rats (male, age 6-8 weeks) were purchased from the experimental animal center in the Guangzhou university of Chinese medicine. They were randomly assigned to receive oral gavage for 5 days with normal saline $(\mathrm{n}=10)$ or specific concentrations of XYS $(5.256 \mathrm{~g} / \mathrm{kg}$, low, $\mathrm{n}=10 ; 10.053 \mathrm{~g} / \mathrm{kg}$, medium, $\mathrm{n}=10$; $20.106 \mathrm{~g} / \mathrm{kg}$, high, $\mathrm{n}=10$ ). Then, the serum was collected and processed according to the standard operation for the subsequent experiments. Experiments were performed under a project license (No. 20190228113) granted by institutional ethics board of Guangzhou University of Chinese Medicine. All animal experiments were performed according to the guidelines of the Institutional Animal Care and Use Committee of Guangzhou University of Chinese Medicine.

\section{Isolation of macrophages}

To separate PMs, 3 SD rats (male, 6-8 weeks) were anaesthetized and sacrificed. The peritoneal membrane was obtained from under the abdominal musculature. Ice cold phosphate-buffered saline (PBS) was injected into the peritoneal cavity and peritoneum was gently and completely 
massaged. Then, PBS was extracted from peritoneal cavity, and peritoneal cells were collected by centrifugation and purified by fluorescence-activated cell sorting (FACS) by selecting F4/80 positive and CD11c negative populations. The harvested cells were cultured with complete Dulbecco's modified eagle medium (DMEM) with 10\% fetal bovine serum (FBS) (Life Technologies, Waltham, MA, USA) and $10 \mathrm{ng} / \mathrm{mL}$ macrophage colony-stimulating factor (M-CSF) (R\&D Systems, Minneapolis, MN, USA) in 6-well plates for $24 \mathrm{~h}$. The cells were collected by trypsin $(0.5 \%$, from Life Technologies, Waltham, MA, USA). To simulate the stress model, the collected PMs were treated with $10 \mu \mathrm{mol} / \mathrm{L}$ CORT and $50 \mu \mathrm{g} / \mathrm{mL}$ ox-LDL in DMEM or normal DMEM (as the control). Additionally, to explore the function of XYS on CORT-induced stress in PMs, the CORT-stimulated PMs were treated with serum after using XYS (low, medium, high) and then other experiments were performed.

\section{Cell Counting Kit-8 (CCK-8) assay}

Treated PMs were plated in 96-well plates and cultured in incubator. After 12, 24, 48, and $72 \mathrm{~h}, 10 \mu \mathrm{L}$ of CCK-8 solution was added to each well of the plate and incubated for $1 \mathrm{~h}$ respectively. Then, absorbance at $450 \mathrm{~nm}$ was detected by microplate reader.

\section{Oil-Red-O staining}

Treated PMs were plated in chambers and cultured in incubators. After $48 \mathrm{~h}$, the medium was removed, the cells were washed with PBS and fixed with formalin for $15 \mathrm{~min}$. Then, the cells were rinsed with $60 \%$ isopropanol and stained with freshly prepared Oil Red O working solution for $15 \mathrm{~min}$. Subsequently, the cells were rinsed with $60 \%$ isopropanol. Finally, the cells were washed with distilled water and mounted in aqueous mounting medium (Servicebio, Wuhan, China).

\section{Enzyme-linked immunosorbent assay (ELISA) assay}

ELISA kits were used to detect GC and TC levels in the medium. The medium was harvested after treatment, then the GC and TC levels in the medium were detected by ELISA kits (GC kit from Shanghai Mlbio, TC kit from Nanjing Jiancheng Bioengineering) according to the kit instructions.

\section{Reverse transcription (RT)-polymerase chain reaction (PCR) and real-time PCR}

Total RNA from the treated cells was extracted by Trizol reagent (Takara Bio., Kusatsu, Shiga, Japan) and was reversed transcribed into cDNA with DBI PrimeScript RT Reagent Kit. Quantitative real-time PCR (qRTPCR) analysis was examined using DBI SYBR Premix Ex Kit (Takara Bio., Kusatsu, Shiga, Japan). The primers used for the RT-PCR were as follows: GAPDH forward 5'-CCTCGTCTCATAGACAAGATGGT-3', GAPDH reverse 5'-GGGTAGAGTCATACTGGAACATG-3', HSP90 forward 5'-ACTATTGCCAAGTCAGGCAC-3', HSP90 reverse 5'-CCTTTGTTCCACGACCCATT-3', GR forward 5'-ATCCAAAGCCGTTTCACTGT-3', GR reverse 5'-CCAGAAGCCGAAAGTCTGTT-3', ABCA1 forward 5'-GACATCATGGCATTTCTGGC-3', and ABCA1 reverse 5'-TCCTCGCATCCAATAGGTC-3'. GAPDH was used as internal control.

\section{Western blot}

There were about $4 \times 10^{7}$ treated cells washed gently with PBS buffer. After centrifugation, the pellet was resuspended with 5 pellet volumes of cytoplasmic extract buffer $(10 \mathrm{Mm}$ $\mathrm{N}$-2-hydroxyethylpiperazine- $\mathrm{N}$-ethanesulfonic acid (HEPES), $60 \mathrm{Mm} \mathrm{KCl,} 1 \mathrm{Mm}$ ethylenediaminetetraacetic acid (EDTA), 0.075\% NP40, $1 \mathrm{mM}$ dithiothreitol (DTT) and $1 \mathrm{mM}$ phenylmethylsulfonyl fluoride (PMSF), adjusted to $\mathrm{pH}$ 7.6) and were incubated on ice for $3 \mathrm{~min}$. After centrifugation at 1,500 rpms, the superscript was collected to another tube (the cytoplasmic extract). The left pellet was washed with $100 \mathrm{uL}$ cytoplasmic buffer without detergent. After centrifugation, 1 pellet volume nuclear extract buffer ( $20 \mathrm{mM}$ tris(hydroxymethyl)aminomethane (Tris)-Cl, $420 \mathrm{mM} \mathrm{NaCl}, 1.5 \mathrm{mM} \mathrm{MgCl} 2,0.2 \mathrm{mM}$ EDTA, $1 \mathrm{mM}$ PMSF, and $25 \%$ glycerol, adjusted to $\mathrm{pH} 8.0$ ) was added and the pellet was vortexed. Then, the extract was incubated on ice for $10 \mathrm{~min}$. After spinning at maximum speed for $10 \mathrm{~min}$, the superscript was the nucleus extract. The protein concentration was quantified by Pierce BCA protein assay kit (Thermo Fisher Scientific, Waltham, MA, USA). Equal amounts of protein sample were separated by 10 polyacrylamide gels and transferred into polyvinylidene difluoride (PVDF) membrane (Millipore, Burlington, MS, USA) by a $300 \mathrm{~mA}$ current. Then, the membrane was blocked in $5 \%$ non-fat dried milk in tris-buffered saline 
with tween 20 (TBST) overnight at $4{ }^{\circ} \mathrm{C}$ and incubated with the following antibodies: anti-HSP90 (1:1,000), anti-GR (1:500), anti-ABCA1 (1:500), anti-Histone H3 (1:1,000), and anti-glyceraldehyde 3 -phosphate dehydrogenase (GAPDH) $(1: 10,000)$ for $1 \mathrm{~h}$ at room temperature. After washing 3 times with TBST buffer, the membranes were incubated with goat horseradish peroxidase (HRP) antiRabbit IgG or anti-mouse $\lg \mathrm{G}$ for $1 \mathrm{~h}$ at room temperature. Chemiluminescence was used to visualize the immune complex and GAPDH was used as the internal control.

\section{Co-immunoprecipitation (Co-IP)}

The PMs with indicated treatment were collected and resuspended in lysis buffer. Then, the samples were put on ice for $30 \mathrm{~min}$ and centrifuged at max rpm for $30 \mathrm{~min}$ at $4{ }^{\circ} \mathrm{C}$. The supernatant was incubated with protein- $\mathrm{G}$ agarose beads (Invitrogen, Carlsbad, CA, USA) for $2 \mathrm{~h}$ at $4{ }^{\circ} \mathrm{C}$. After centrifugation for $30 \mathrm{~s}$, the supernatant was incubated overnight with rabbit anti-HSP90 antibody at $4{ }^{\circ} \mathrm{C}$. After a series of washes, the protein bound to the beads was eluted by loading buffer followed by incubation in a water bath at $55^{\circ} \mathrm{C}$ for $15 \mathrm{~min}$ and subsequent centrifugation of the protein-G-beads. Finally, samples of the supernatant were analyzed by western blot to observe the combination of GR and HSP90.

\section{GR and ABCA1 expression vector}

The full-length wild type coding sequence (CDS) of GR and ABCA1 were amplified and cloned into the pcDNA3.0 (for GR) and PGL3 (for ABCA1) vectors (Sangon Biotech, Shanghai, China). The primers used in the vector construction were as follows: GR forward 5'-CGGGGTAC CATGGACTCCAAAGAATCCTTAGCT-3', GR reverse 5'-CCGCTCGAGTCATTTTTGATGAAACAGAAG CTTT-3', wild type ABCA1 forward 5'-GGGGTACCT TTTTAGTAAAGACTGGGTTTCATC-3', wild type ABCA1 reverse 5'-CCGCTCGAGGTACTCACATCAG TGGTTCTATCAA-3', mutant ABCA1 forward 5'-ATC CATCCCTTTCTAGCCATGTTACACGGTTGGTG CAAAGGGGCC-3', and mutant ABCA1 reverse 5'-GGC CCCTTTGCACCAACCGTGTAACATGGCTAGAAA GGGATGGAT-3'. These vectors were identified by DNA sequencing and western blot (to detect the indicated protein expression).

\section{Dual-luciferase reporter assay}

The full-length wild type and mutant promotor region of ABCA1 were amplified and cloned into the PGL3 vector. Wild type or mutant vectors were co-transfected with a GR expression vector into the PMs using Lipofectamine 2000 (Invitrogen, Carlsbad, CA, USA). The cells were collected and the luciferase activity was tested $48 \mathrm{~h}$ after the transfection with Dual-luciferase reporter assay system according to the manufacture's protocol (Promega, Madison, WI, USA).

\section{Statistical analysis}

The data are presented as mean \pm standard deviation, and were analyzed by Student's $t$-test. A value of $\mathrm{P}<0.05$ was considered as statically significant.

\section{Results}

\section{XYS increased proliferation and decreased lipids in a CORT-induced stress model of rat PMs}

In order to explore the effect of serum including XYS, we made a CORT-induced stress model in rat PMs and administered a serum including an indicated concentration of XYS (low, medium, high) or normal DMEM as the control. A CCK-8 assay was used to detect the viability of the treated PMs. The data showed CORT-induced stress inhibited proliferation, while serum including XYS dosedependently increased the viability compared with the CORT treated PMs (Figure 1A). Using an oil red staining assay to detect the level of lipids in the cells, it was revealed that CORT-induced stress increased lipid accumulation in PMs, and serum including XYS could dose-dependently decrease the CORT-induced lipid accumulation in PMs (Figure 1B,C). Therefore, our results suggested serum including XYS exerted a protective function in CORTinduced stress PMs.

\section{XYS increased ABCA1 and decreased GR expression levels in the CORT-induced stress rat model of PMs}

Furthermore, ELISA assay was used to detect GC and TC levels in treated PMs. Our results revealed CORT-induced stress could increase GC and TC levels in PMs (Figure 2A,B). Serum including XYS could decrease the TC level while it 
A

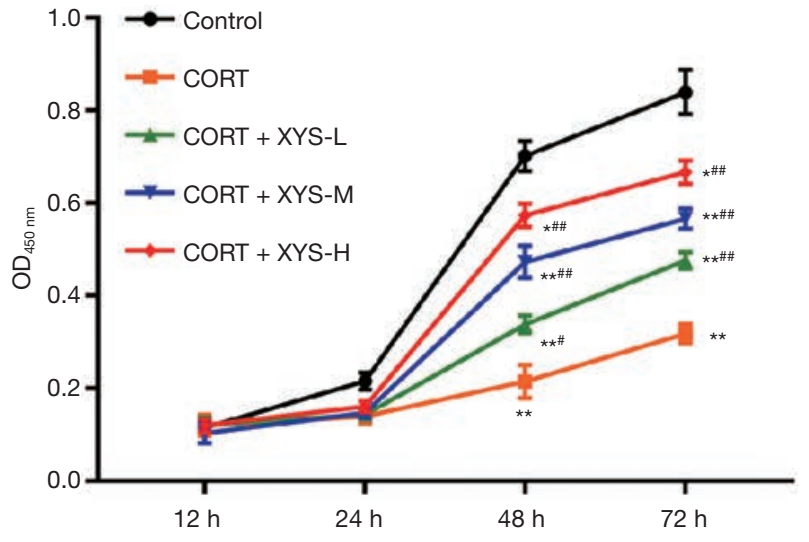

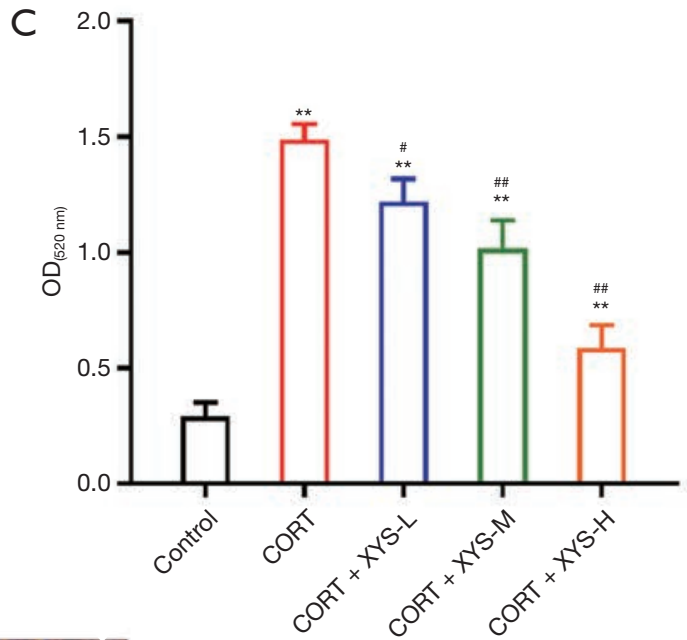

B Control
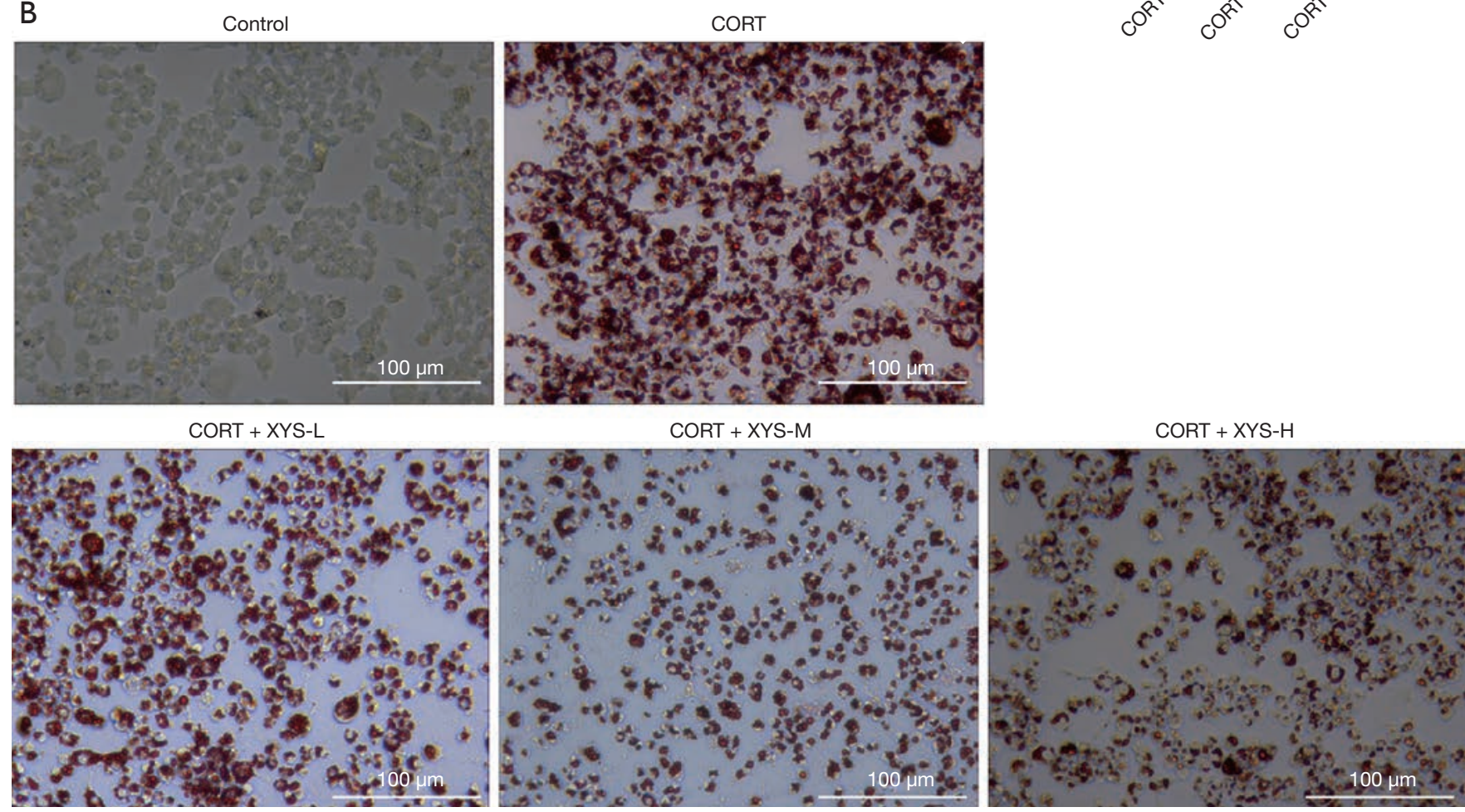

Figure 1 Serum including XYS increases proliferation and decreases lipids in CORT-induced stress model of rat PMs. Rat PMs were treated with CORT to construct acute stress model (treated with normal DMEM as the control). Then, CORT-treated PMs were given serum including indicated XYS (low, medium and high) following the other examination. (A) CCK-8 assay was used to detect the viability of the CORT-induced stress rat PMs treated with serum including indicated XYS. *, $\mathrm{P}<0.05$, ${ }^{* *}, \mathrm{P}<0.01$ vs. Control; ${ }^{\#}, \mathrm{P}<0.05$, ${ }^{\#}, \mathrm{P}<0.01$ vs. CORT. (B,C) Oil red staining was used to test the lipids accumulation in these treated PMs, and the relative quantity of OD value was also calculated. **, $\mathrm{P}<0.01$ vs. Control; ${ }^{*}, \mathrm{P}<0.05,{ }^{\# \#, ~} \mathrm{P}<0.01$ vs. CORT. XYS, Xiaoyaosan; PMs, peritoneal macrophages; CORT, corticosterone; DMEM, Dulbecco's modified eagle medium; OD, optical density.

had no effect on the level of GC in CORT-induced stress PMs (Figure $2 A, B)$. A previous publication showed the receptor of GC (GR), like many other signaling receptors, depends on HSP90 to play its function, and, HSP90 works as molecular chaperone. The ABCA1 protein is a kind of transporter associated with accumulation of lipids. Therefore, we detected expression levels of HSP90, ABCA1, and GR. The results of RT-PCR showed CORT-induced 


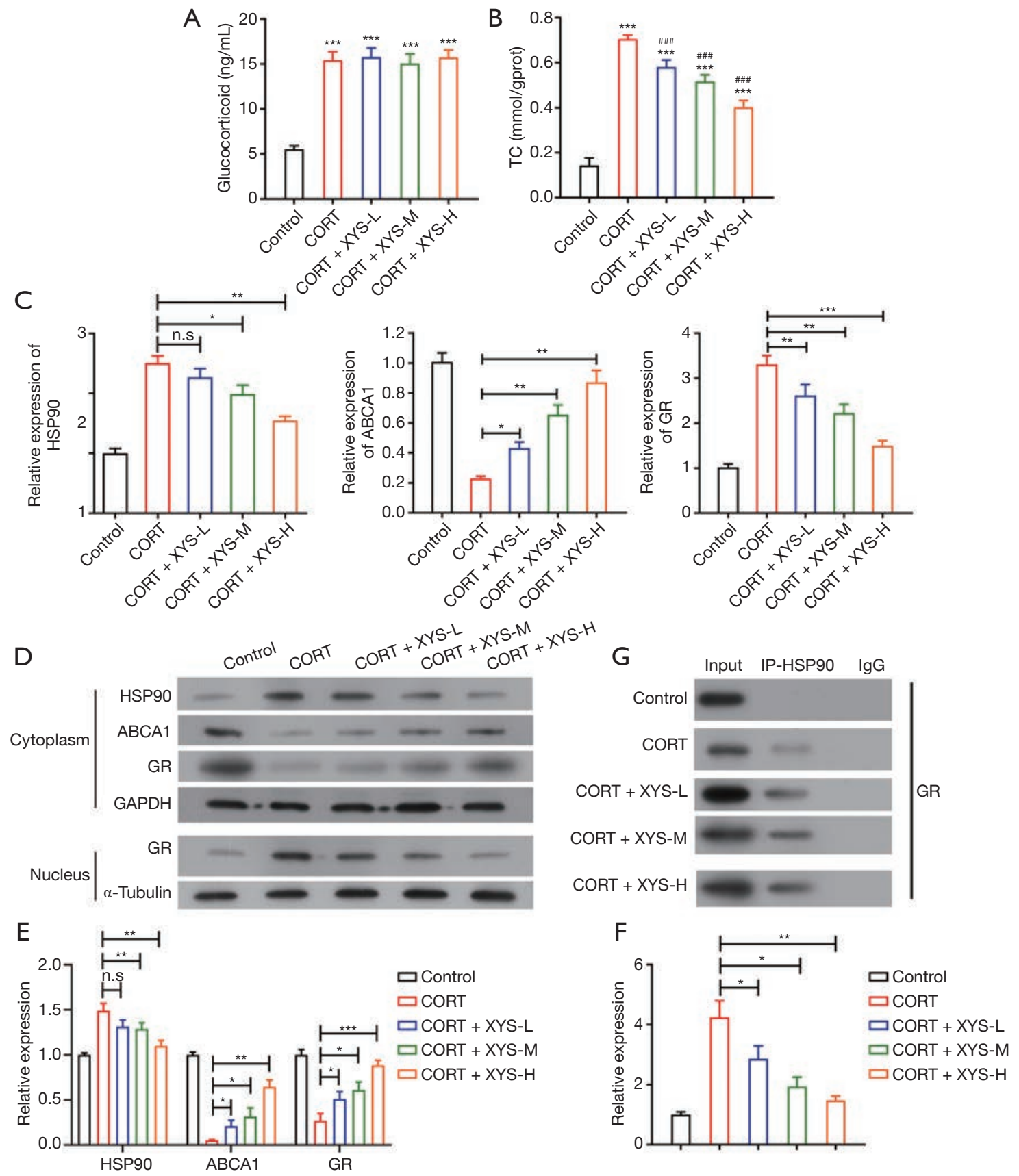

Figure 2 Serum including XYS increased ABCA1 protein level in cytoplasm and decreased GR protein level in nucleus in CORT-induced stress rat model of PMs. ELISA was used to detect GC (A) and TC (B) level in the medium of PMs with indicated treatment, *** $\mathrm{P}<0.001$ vs. control; ${ }^{\# \#}, \mathrm{P}<0.001$ vs. CORT. (C) RT-PCR was used to test the mRNA expression level of HSP90, ABCA1 and GR, *, P<0.05, **, $\mathrm{P}<0.01,{ }^{* * *}, \mathrm{P}<0.001$ vs. CORT. (D,E,F) Western blot was used to detect HSP90, GR and ABCA1 protein level in cytoplasm and GR protein expression level in nucleus, for the cytoplasm, GAPDH was used as the internal control and for the nucleus, $\alpha$-Tubulin was used as internal control (D), (E) was the quantified protein expression level in cytoplasm and (F) was the nucleus level, n.s, no significant difference. *, $\mathrm{P}<0.05,{ }^{* *}, \mathrm{P}<0.01$, ***, $\mathrm{P}<0.001$ vs. CORT. (G) To further investigate the relevance between HSP90 and GR, we used HSP90 to pull down GR and the result showed CORT-induced stress decreased the interaction of HSP90 and GR, and serum including XYS could increase their interaction. XYS, Xiaoyaosan; PMs, peritoneal macrophages; CORT, corticosterone; RT-PCR, real time polymerase chain reaction; GAPDH, glyceraldehyde 3-phosphate dehydrogenase; GR, glucocorticoid receptor; ELISA, enzyme-linked immunosorbent assay. 
stress suppressed the mRNA level of ABCA1 and elevated mRNA expression level of HSP90 and GR (Figure 2C). Serum including XYS could dose-dependently increase ABCA1 mRNA expression level and decrease HSP90 and GR mRNA level in CORT-induced stress PMs (Figure 2C). Further analysis showed serum including XYS could decrease the HSP90 protein level in cytoplasm and increase ABCA1 and GR protein expression levels in cytoplasm (Figure 2D,E,F), while decreasing the GR protein level in nucleus (Figure 2D,E,F). These results indicated HSP90 might interact with GR in CORT-induced stress PMs. To further investigate the relevance between HSP90 and GR, we used HSP90 to pull down GR and the result showed CORT-induced stress decreased the interaction of HSP90 and GR, and serum including XYS could increase their interaction (Figure $2 G$ ).

\section{Serum including XYS combined with ABCA1 and GR reversed the function of CORT on cell viability and accumulation of lipids}

After having shown that serum including XYS could dose-dependently increase the viability and decrease lipid accumulation CORT-induced stress PMs (Figure 1), for subsequent study we just selected the serum with a medium concentration of XYS which also performed a significant function. The CCK-8 assay showed only serum including XYS combined with overexpression of ABCA1 and elevated GR could reverse the effect of CORT-induced inhibition of viability in PMs (Figure 3A). Similarly, the oil red staining revealed only serum including XYS combined with overexpression of ABCA1 and elevated GR could strongly reduce accumulation of lipids in CORT-induced stress PMs (Figure 3B,C).

\section{ABCA1 exerted its function by binding to GR in CORT- induced stress rat PMs}

To further study how ABCA1 performed its function, we detected the level of TC in PMs. ELISA result showed both GR or GR combined with ABCA1 could reduce CORTinduced TC accumulation in PMs (Figure 4A). Besides, the combination of GR and ABCA1 was obviously stronger at inhibiting TC accumulation compared with GR alone in the stressed PMs (Figure 4A). The RT-PCR results showed elevated GR inhibited the ABCA1 mRNA level (Figure 4B) and overexpression of ABCA1 had no effect on the mRNA level of GR (Figure 4C) in CORT-induced stress PMs. In addition, the western blot data was consistent with the PCR results (Figure 4C,D,E), which suggested that ABCA1 might be controlled by GR. To further investigate the interaction of ABCA1 and GR, we constructed a wild type and mutant (binding site) ABCA1 luciferase reporter vector. The GR and wild type (or mutant) ABCA1 reporter vectors were transfected into PMs; then, luciferase activity was tested, and the data showed GR could increase the luciferase activity of wild type ABCA1 vector and had no effect on mutant vector (Figure $4 F$ ). In summation, our results suggested ABCA1 might exert its function by binding to GR in CORT-induced stress PMs.

\section{Discussion}

The traditional Chinese medicine XYS is mainly used in the treatment of depressive diseases as monotherapy or combined with other agents in China $(19,20)$. However, a report also found that XYS could influence body weight by regulating expression of leptin and leptin receptors (21). In addition, XYS decreased the level of serum CORT and had a suppressive effect on CORT-induced stress $(22,23)$. To explore whether XYS could suppress the lipid accumulation induced by CORT, we generated an ox-LDL and CORTinduced stress model in rat PMs following the treatment of XYS-containing serum. Our results showed that CORTinduced stress inhibited proliferation and increased lipid accumulation in PMs, which was similar to the results of previous studies $(24,25)$. We also found that serum including XYS could dose-dependently increase proliferation, decrease lipid accumulation, and elevate TC level in CORT-induced PMs. In a clinical study, it was also found that the TC level was decreased in an obese type 2 diabetes patient group receiving metformin and XYS treatment (26). These results suggested that XYS could exert a protective function for CORT-induced stress macrophages.

A previous study revealed XYS markedly inhibited the nuclear translocation of GR and increased its expression at the mRNA and protein level in chronic psychological stress rats' hippocampal neuron cells (27). In this study, we also showed that XYS decreased GR protein expression in the nucleus and GR mRNA expression in CORT-induced stress rat PMs. These data suggested XYS could protect PMs from CORT-induced stress by inhibiting the elevated GR protein level from being transported into the nucleus. Previous reports also showed that HSP90 could assist the internuclear transfer of GC-GR complex, then GC-GR complex could bind to the GR element (GRE) and induce 

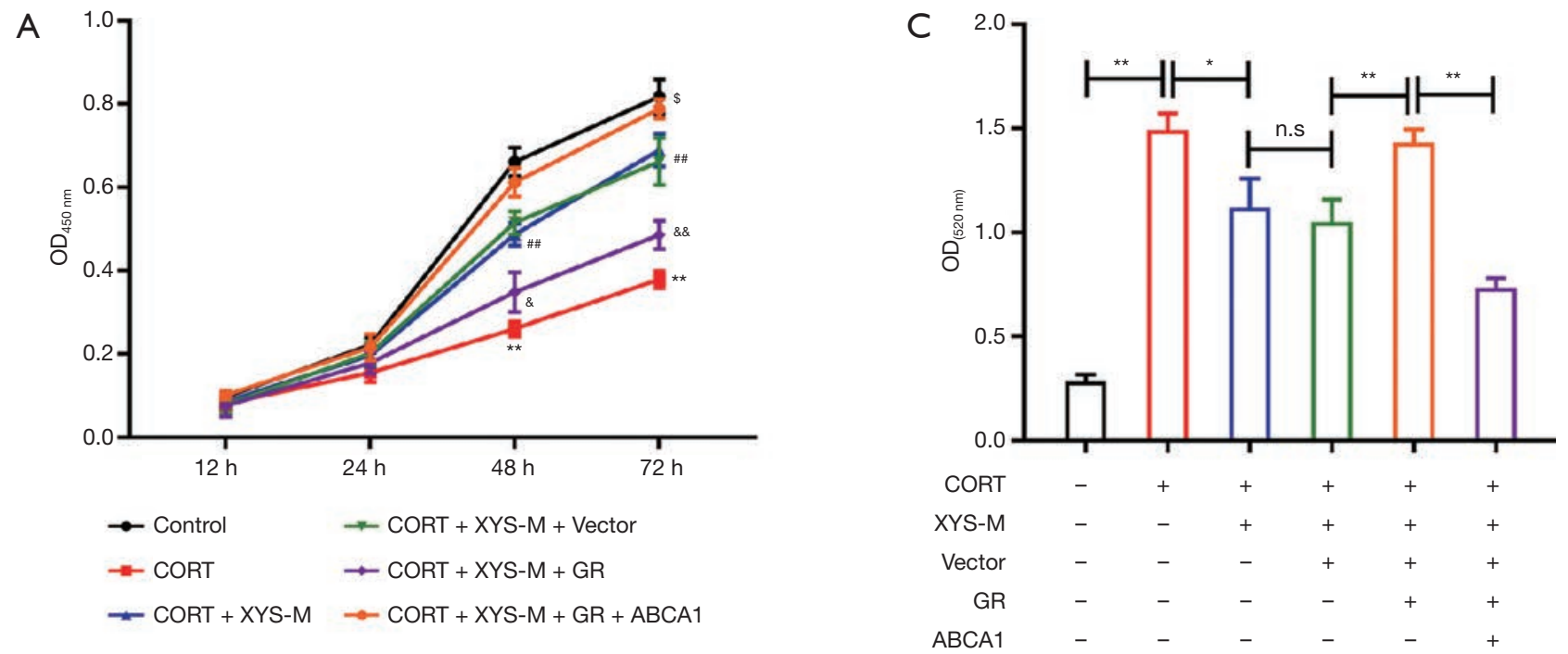

B Control CORT
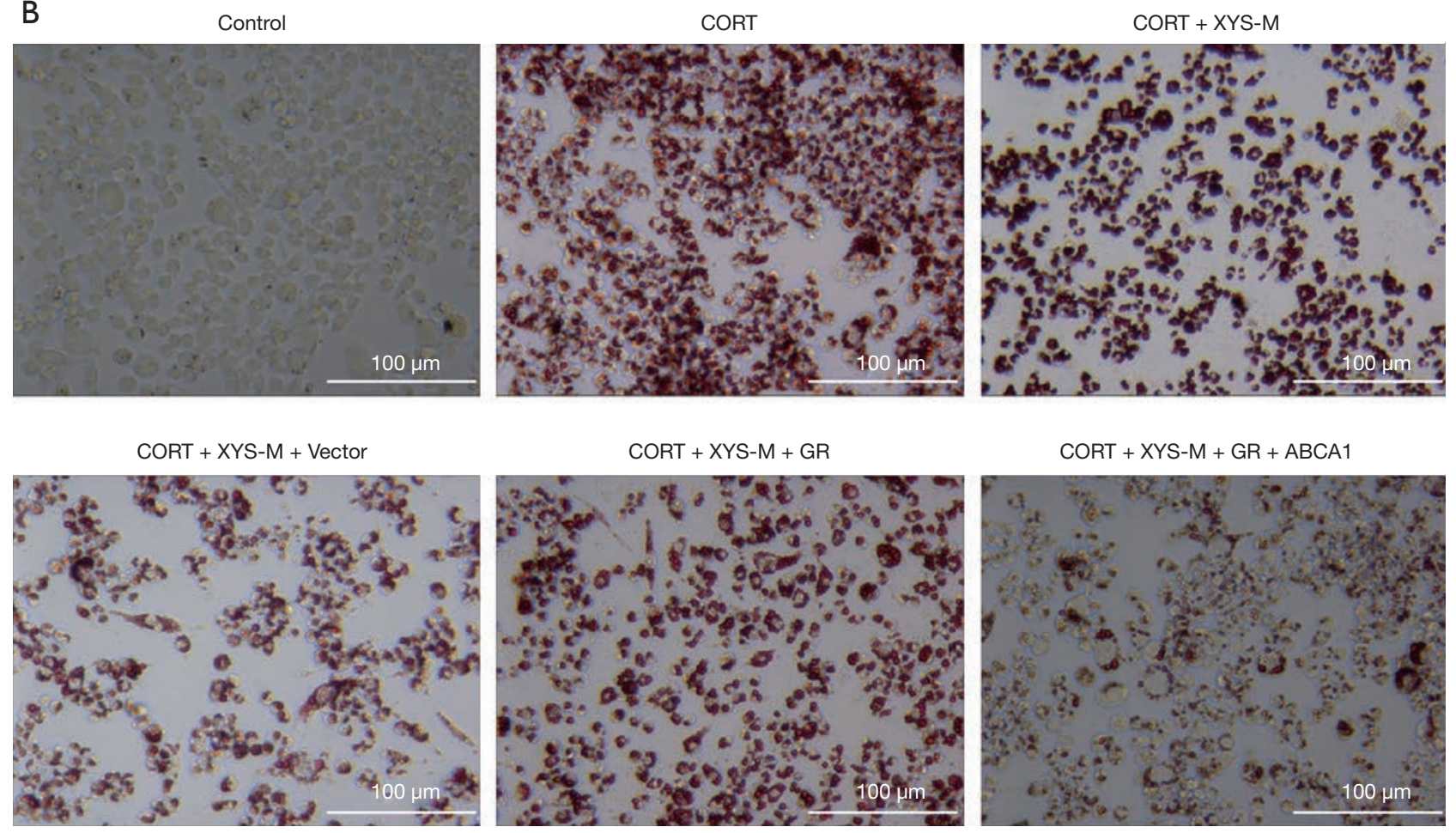

Figure 3 Serum including XYS combined with ABCA1 and GR reversed the function of CORT on viability and lipids accumulation. CORT-treated PMs with serum including medium XYS were transfected with ABCA1 or/and GR expression vector following the other experiments. (A) CCK-8 assay was used to detect the viability of the $\mathrm{PMs}$ with the indicated treatment. ${ }^{* *}, \mathrm{P}<0.01$ vs. Control; ${ }^{\# \#}$, $\mathrm{P}<0.01$ vs. CORT; ${ }^{\$}, \mathrm{P}<0.05$ vs. CORT + XYS-M + Vector; ${ }^{\&}, \mathrm{P}<0.05,{ }^{\& \&}, \mathrm{P}<0.01$ vs. CORT + XYS-M + GR. (B) Oil red staining was used to test the lipid accumulation in the PMs with indicated treatment. (C) The results of Oil red staining were also counted based on the OD value. *, $\mathrm{P}<0.05$, ** $\mathrm{P}<0.01$, n.s, no significant. XYS, Xiaoyaosan; PMs, peritoneal macrophages; CORT, corticosterone; GR, glucocorticoid receptor; OD, optical density. 

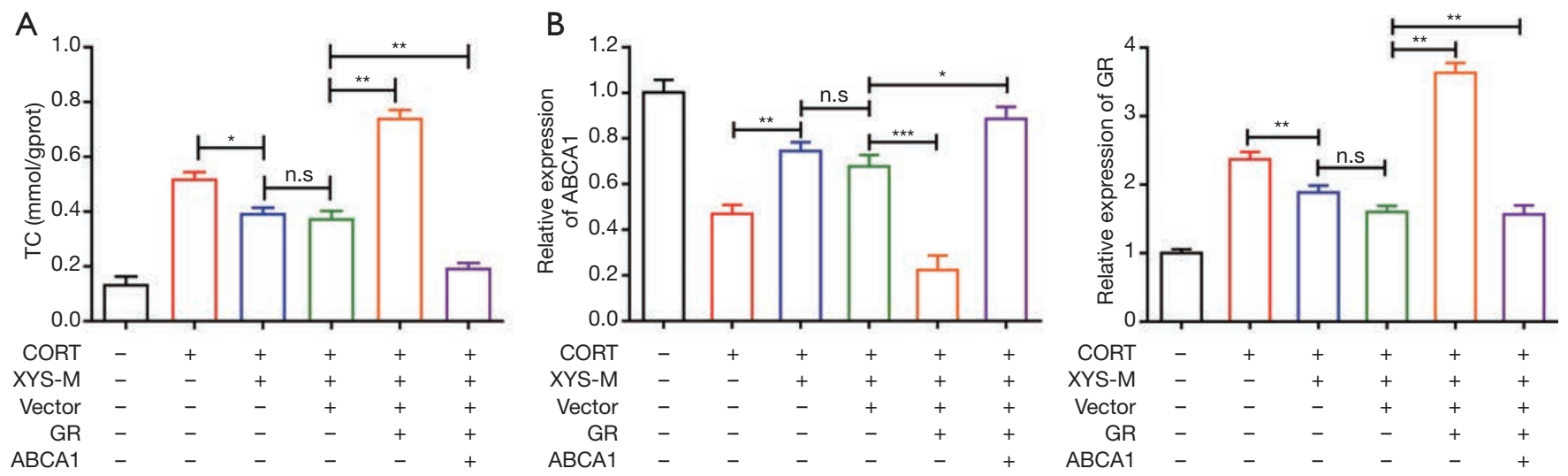

C

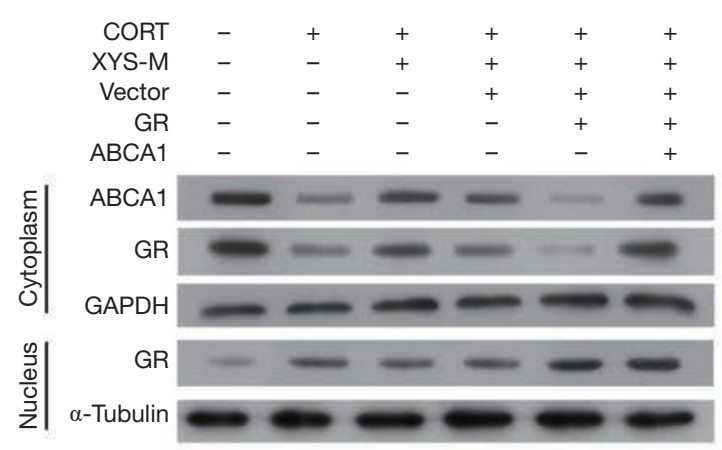

$\mathrm{E}$

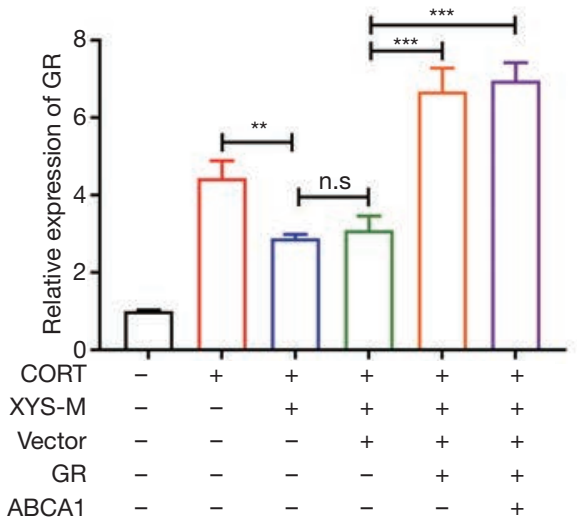

D

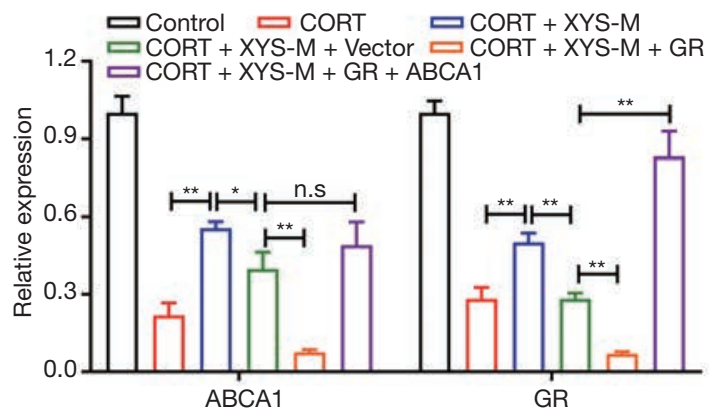

$\mathrm{F}$
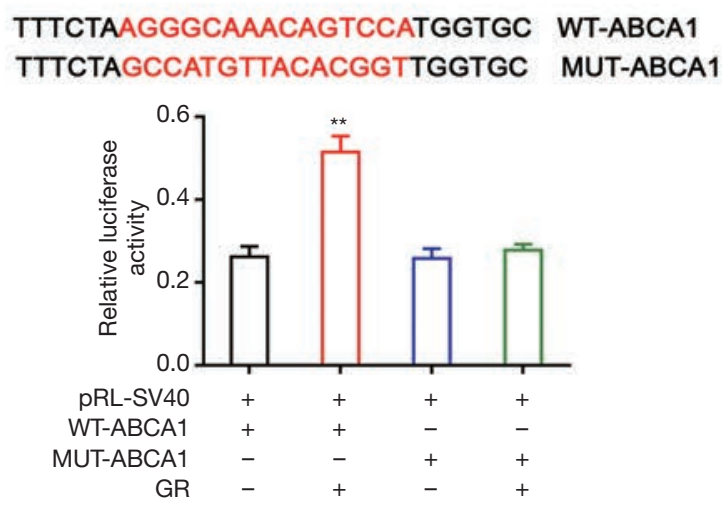

Figure 4 ABCA1 exerted its function by binding to GR in CORT-induced stress rat PMs. (A) ELISA was used to examine the TC level in PMs with indicated treatment. *, $\mathrm{P}<0.05$; ${ }^{*}, \mathrm{P}<0.01$. (B) RT-PCR was used to test ABCA1 and GR mRNA expression level in PMs with indicated treatment, n.s, no significant difference. *, $\mathrm{P}<0.05$; ${ }^{* *}, \mathrm{P}<0.01$; ${ }^{* *}, \mathrm{P}<0.001$. (C,D,E) Western blot was used to test ABCA1 and GR protein expression level in cytoplasm and nucleus of PMs with indicated treatment; for the cytoplasm, GAPDH was used as the internal control; for the nucleus, $\alpha$-Tubulin was used as internal control (C), (D) (cytoplasm) and (E) (nucleus) were the quantified results. **, $\mathrm{P}<0.01$; ***, $\mathrm{P}<0.001$. (F) Wild type and mutant CDS of ABCA1 were cloned to pRL-SV40. GR and wild type (or mutant) ABCA1 reporter vectors were con-transfected to PMs, then, luciferase activity was detected, upper lane was the part sequence of wild type and mutant ABCA1, lower lane was the quantified result. ${ }^{* *}, \mathrm{P}<0.01$ vs. pRL-SV40 + WT-ABCA1. n.s, no significant difference; PMs, peritoneal macrophages; CORT, corticosterone; GR, glucocorticoid receptor; RT-PCR, real time polymerase chain reaction; ELISA, enzyme-linked immunosorbent assay; GAPDH, glyceraldehyde 3-phosphate dehydrogenase. 


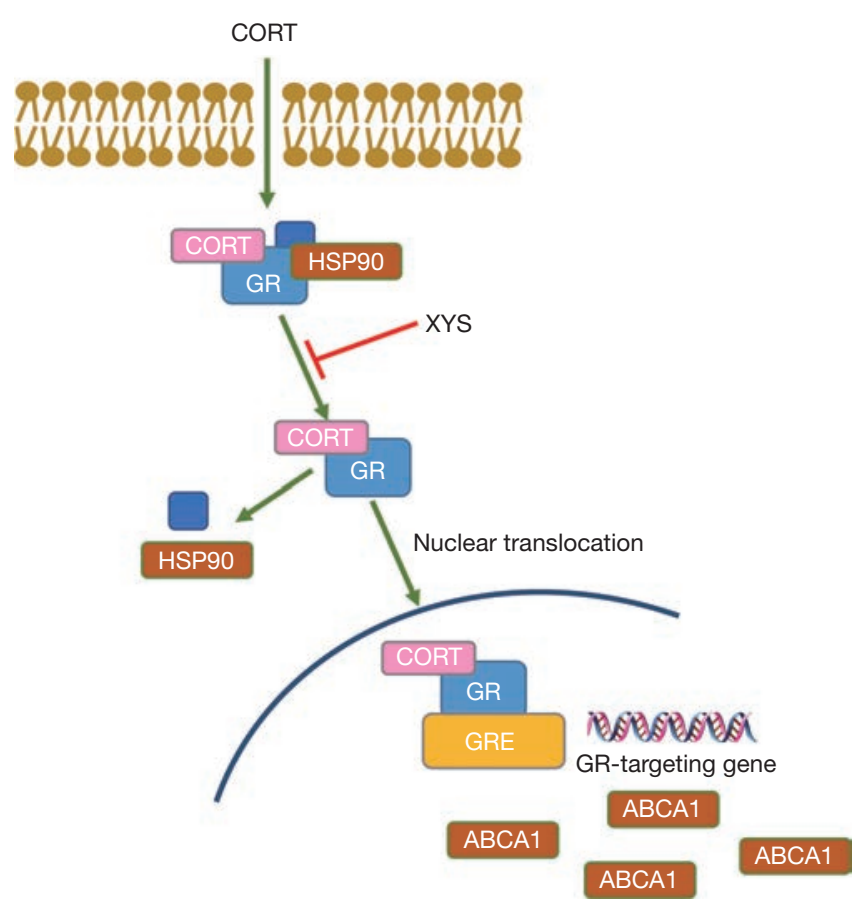

Figure 5 A schematic diagram describing the underlying regulatory mechanism of XYS on CORT-induced stress rat PMs. CORT enhanced the dissociation of GR and HSP90 protein complex, whereas XYS treatment reduced the dissociation of the protein complex. As a result, XYS decreased GR protein level that transported into the nucleus through the nuclear pores, leading to the decline of GR binding to glucocorticoid response element (GRE) and the elevation of its target gene ABCA1. Consequently, XYS played a protective role on CORT-induced stress rat PMs. XYS, Xiaoyaosan; PMs, peritoneal macrophages; CORT, corticosterone; GR, glucocorticoid receptor.

the expression of the target genes $(13,28)$. When released from HSP90, the activity of GR's transportation into the nucleus was enhanced. Our results showed XYS could promote the protein interaction of GR and HSP90 in the cytoplasm of CORT-induced PMs, which indicated XYS performed its protective function by reducing GR protein levels in the nucleus.

In this study, we showed serum including XYS could dose-dependently decrease GR expression in nucleus, while increasing ABCA1 protein level in the cytoplasm of PMs. A member of ATP-binding cassette transporters, ABCA1 is associated with the level of circulating HDL and accumulation of $\mathrm{CE}$ in various tissues, which is mainly distributed in macrophage-derived foam cells (29-31). Previously, they showed GR ligands could affect ABCA1 expression and cholesterol efflux from macrophages which were regulated by GR through a LXR-independent manner (32). Regulation of ABCA1 expression and apolipoproteinmediated cholesterol efflux from macrophages could be controlled by GR (33). Our further study showed GR elevated by overexpression decreased the ABCA1 level, while overexpression of ABCA1 had no effect on the expression level of GR, which indicated that XYS could promote lipid efflux in CORT-induced PMs through GRregulated $\mathrm{ABCA} 1$. To further investigate the relationship between ABCA1 and GR, we constructed a wild type and mutant ABCA1 luciferase reporter. Our results revealed GR could increase the luciferase activity of wild type ABCA1 but had no effect on the luciferase activity of mutant ABCA1 reporter, which suggested GR could regulate expression of ABCA1 by banding to its 5'-non-coding sequence. As lipid accumulation in macrophages plays an important role in atherosclerosis $(34,35)$, we could speculate that XYS might have a therapeutic effect on atherosclerosis.

\section{Conclusions}

In summary, we found that XYS-containing serum increased viability and decreased lipid accumulation in CORTinduced stress rat PMs. The application of XYS decreased expression of HSP90 protein and increased expression of ABCA1 protein in cytoplasm, while it decreased GR protein in nucleus. Our study suggested XYS might exert its function by enhancing the interaction of HSP90 and GR (Figure 5). These results broaden the understanding of the function and mechanism of XYS in CORT-induced stress in macrophages.

\section{Acknowledgments}

Funding: This work was supported by the National Natural Science foundation of China (81573922), Sanming Project of Medicine in the Shenzhen-Chinese Academy of Medical Sciences Fuwai Hospital Professor Zhang Jian Cardiovascular Disease Team (SZSM201612033), and Shenzhen Municipal Scheme for Basic Research (JCYJ20190808144005614).

\section{Footnote}

Reporting Checklist: The authors have completed the ARRIVE reporting checklist. Available at http://dx.doi. org/10.21037/atm-20-6505 
Data Sharing Statement: Available at http://dx.doi. org/10.21037/atm-20-6505

Conflicts of Interest: All authors have completed the ICMJE uniform disclosure form (available at http://dx.doi. org/10.21037/atm-20-6505). The authors have no conflicts of interest to declare.

Ethical Statement: The authors are accountable for all aspects of the work in ensuring that questions related to the accuracy or integrity of any part of the work are appropriately investigated and resolved. Experiments were performed under a project license (No. 20190228113) granted by institutional ethics board of Guangzhou University of Chinese Medicine. All animal experiments were performed according to the guidelines of the Institutional Animal Care and Use Committee of Guangzhou University of Chinese Medicine.

Open Access Statement: This is an Open Access article distributed in accordance with the Creative Commons Attribution-NonCommercial-NoDerivs 4.0 International License (CC BY-NC-ND 4.0), which permits the noncommercial replication and distribution of the article with the strict proviso that no changes or edits are made and the original work is properly cited (including links to both the formal publication through the relevant DOI and the license). See: https://creativecommons.org/licenses/by-nc-nd/4.0/.

\section{References}

1. Wang M, Huang W, Gao T, et al. Effects of Xiao Yao San on interferon-alpha-induced depression in mice. Brain Res Bull 2018;139:197-202.

2. Zhao HB, Jiang YM, Li XJ, et al. Xiao Yao San Improves the Anxiety-Like Behaviors of Rats Induced by Chronic Immobilization Stress: The Involvement of the JNK Signaling Pathway in the Hippocampus. Biol Pharm Bull 2017;40:187-94.

3. Yan ZY, Li XJ, Ding XF, et al. Evaluating the Antidepression Effect of Xiaoyaosan on Chronically-stressed Mice. J Vis Exp 2019. doi: 10.3791/58276.

4. Jiao H, Yan Z, Ma Q, et al. Influence of Xiaoyaosan on depressive-like behaviors in chronic stress-depressed rats through regulating tryptophan metabolism in hippocampus. Neuropsychiatr Dis Treat 2018;15:21-31.

5. Lun Z. A study on the antidepressant effect of Danzhi Xiaoyao Powder. Afr J Tradit Complement Altern Med
2013;11:205-8.

6. Luo HG, Chen JX, Zhang Q, et al. Comparative study on effects of Xiaoyao Powder and its modified prescription on plasma metabolomics of rats with chronic immobilization stress. Chin J Integr Med 2013;19:610-5.

7. Ding J, Chen JX, Liang Y, et al. Effect of Xiaoyaosan on blood ACTH and CORT levels of rat with liver depression and spleen deficiency syndrome. China Journal of Traditional Chinese Medicine \& Pharmacy 2009;24:1436-9.

8. Okutsu M, Lira VA, Higashida K, et al. Corticosterone accelerates atherosclerosis in the apolipoprotein E-deficient mouse. Atherosclerosis 2014;232:414-9.

9. Uchoa ET, Aguilera G, Herman JP, et al. Novel aspects of glucocorticoid actions. J Neuroendocrinol 2014;26:557-72.

10. Bai Z, Li H, Zheng K, et al. A good response to glucocorticoid for sub-acute liver failure: a case report. Transl Gastroenterol Hepatol 2018;3:25.

11. Desmet SJ, De Bosscher K. Glucocorticoid receptors: finding the middle ground. J Clin Invest 2017;127:1136-45.

12. Chen H, Xing J, Hu X, et al. Inhibition of heat shock protein 90 rescues glucocorticoid-induced bone loss through enhancing bone formation. J Steroid Biochem Mol Biol 2017;171:236-46.

13. Morishima Y, Mehta RK, Yoshimura M, et al. Chaperone Activity and Dimerization Properties of Hsp90 $\alpha$ and Hsp90 $\beta$ in Glucocorticoid Receptor Activation by the Multiprotein Hsp90/Hsp70-Dependent Chaperone Machinery. Mol Pharmacol 2018;94:984-91.

14. Lasker MV, Leventhal SM, Lim D, et al. Hyperactive Human Glucocorticoid Receptor Isoforms and Their Implications for the Stress Response. Shock 2015;43:228.

15. Cho W, Kang JL, Park YM. Corticotropin-Releasing Hormone (CRH) Promotes Macrophage Foam Cell Formation via Reduced Expression of ATP Binding Cassette Transporter-1 (ABCA1). PLoS One 2015;10:e0130587.

16. Adorni MP, Favari E, Ronda N, et al. Free cholesterol alters macrophage morphology and mobility by an ABCA1 dependent mechanism. Atherosclerosis 2011;215:70-6.

17. Sun L, Li E, Wang F, et al. Quercetin increases macrophage cholesterol efflux to inhibit foam cell formation through activating PPARgamma-ABCA1 pathway. Int J Clin Exp Pathol 2015;8:10854-60.

18. Zhang M, Li L, Xie W, et al. Apolipoprotein A-1 binding protein promotes macrophage cholesterol efflux by facilitating apolipoprotein A-1 binding to ABCA1 and preventing $\mathrm{ABCA1}$ degradation. Atherosclerosis 
2016;248:149-59.

19. Sun HY, Li Q, Liu YY, et al. Xiao-Yao-San, a Chinese Medicine Formula, Ameliorates Chronic Unpredictable Mild Stress Induced Polycystic Ovary in Rat. Front Physiol 2017;8:729.

20. Cao GP, Gui D, Fu LD, et al. Anxiolytic and neuroprotective effects of the Traditional Chinese Medicinal formulation Dan-zhi-xiao-yao-san in a rat model of chronic stress. Mol Med Rep 2016;14:1247-54.

21. Wang SX, Chen JX, Yue GX, et al. Xiaoyaosan Decoction Regulates Changes in Neuropeptide Y and Leptin Receptor in the Rat Arcuate Nucleus after Chronic Immobilization Stress. Evid Based Complement Alternat Med 2012;2012:381278.

22. Yang J, Gong X, Liu R, et al. Effect of the decoction of Xiaoyaosan and its efficacy drug-groups on the level of serum cytokines and corticosterone in CUMS model rats. Pharmacology \& Clinics of Chinese Materia Medica 2013;29:4-6.

23. Wu LL, Ran CL, Yan C, et al. Effect of Xiaoyaosan on proliferation and differentiation of hippocampus nerve precursor cells under high corticosterone condition. Chinese Journal of Pathophysiology 2009;25:97-103.

24. Nakatani Y, Amano T, Takeda H. Corticosterone suppresses the proliferation of RAW264.7 macrophage cells via glucocorticoid, but not mineralocorticoid, receptor. Biol Pharm Bull 2013;36:592-601.

25. Yuan N, Gong L, Tang K, et al. An Integrated Pharmacology-Based Analysis for Antidepressant Mechanism of Chinese Herbal Formula Xiao-Yao-San. Front Pharmacol 2020;11:284.

26. Li XZ. Clinical effect of using metformin and Xiaoyaosan for the treatment of obese type 2 diabetes. Chinese Journal of Clinical Rational Drug Use 2018.

27. Yu S, Fu L, Lu J, et al. Xiao-Yao-San reduces blood-brain barrier injury induced by chronic stress in vitro and vivo

Cite this article as: Chen $M$, Huang $\mathrm{R}, \mathrm{Fu} W, \mathrm{Ou} L$, Men L, Zhang Z, Yang S, Liu Q, Luan J. Xiaoyaosan (Tiaogan-Liqi therapy) protects peritoneal macrophages from corticosterone-induced stress by regulating the interaction between glucocorticoid receptor and ABCA1. Ann Transl Med 2020;8(22):1506. doi: 10.21037/atm-20-6505 via glucocorticoid receptor-mediated upregulation of Occludin. J Ethnopharmacol 2020;246:112165.

28. Zhao Y, Xiong RP, Chen X, et al. Hsp90 regulation affects the treatment of glucocorticoid for pancreatitis-induced lung injury. Mol Cell Biochem 2018;440:189-97.

29. Franssen R, Schimmel AW, van Leuven SI, et al. In Vivo Inflammation Does Not Impair ABCA1-Mediated Cholesterol Efflux Capacity of HDL. Cholesterol 2012;2012:610741.

30. Zhao Y, Pennings M, Hildebrand RB, et al. Enhanced foam cell formation, atherosclerotic lesion development, and inflammation by combined deletion of ABCA1 and SR-BI in Bone marrow-derived cells in LDL receptor knockout mice on western-type diet. Circ Res 2010;107:e20-31.

31. Yin K, Liao DF, Tang CK. ATP-binding membrane cassette transporter A1 (ABCA1): a possible link between inflammation and reverse cholesterol transport. Mol Med 2010;16:438-49.

32. Fu W, Chen M, Ou L, et al. Xiaoyaosan prevents atherosclerotic vulnerable plaque formation through heat shock protein/glucocorticoid receptor axis-mediated mechanism. Am J Transl Res 2019;11:5531-45.

33. Islam RM, Pourmousa M, Sviridov D, et al. Structural properties of apolipoprotein A-I mimetic peptides that promote ABCA1-dependent cholesterol efflux. Sci Rep 2018;8:2956.

34. Miao H. microRNA-212 promotes lipid accumulation and attenuates cholesterol efflux in THP-1 human macrophages by targeting SIRT1. Gene 2018;643:55.

35. Hulsmans M, Sager HB, Roh JD, et al. Cardiac macrophages promote diastolic dysfunction. J Exp Med 2018;215:423-40.

(English Language Editor: J. Jones) 